\title{
Toints
}

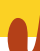

\section{High tibial osteotomy in varus knees: indications and limits}

\author{
MARCO CORGIAT LOIA, STEFANIA VANNI, FEDERICA ROSSO, DAVIDE EDOARDO BONASIA, \\ MATTEO BRUZZONE, FEDERICO DETTONI, ROBERTO ROSSI
}

Department of Orthopaedics and Traumatology, Ospedale Mauriziano, Torino, Italy

\begin{abstract}
Opening wedge high tibial osteotomy (OWHTO) is a surgical procedure that aims to correct the weight-bearing axis of the knee, moving the loads laterally from the medial compartment. Conventional indications for OWHTO are medial compartment osteoarthritis and varus malalignment of the knee; recently OWHTO has been used successfully in the treatment of double and triple varus. OWHTO, in contrast to closing wedge high tibial osteotomy, does not require fibular osteotomy or peroneal nerve dissection, or lead to disruption of the proximal tibiofibular joint and bone stock loss. For these reasons, interest in this procedure has grown in recent years.

The aim of this study is to review the literature on OWHTO, considering indications and prognostic factors (body mass index, grade of osteoarthritis, instability, range of movement and age), outcomes at midterm follow-up, and limits of the procedure (slope modifications, patellar height changes and difficulties in conversion to a total knee arthroplasty).
\end{abstract}

Keywords: high tibial osteotomy, knee, osteoarthritis, opening wedge, prognostic factors.

\section{Introduction}

High tibial osteotomy (HTO) is a widely accepted procedure for treating varus alignment of the knee associated with medial compartment overload/osteoarthritis

\footnotetext{
Corresponding Author:

Marco Corgiat Loia, MD

Department of Orthopaedics and Traumatology

Ospedale Mauriziano

Largo Turati 62, 10126 Torino, Italy

E-mail: marco.corgiat@hotmail.it
}

$(\mathrm{OA})$. The purpose of the procedure, which may be a medial opening wedge, lateral closing wedge, dome or "en chevron" osteotomy, is to shift the mechanical axis of the lower limb from the medial to the lateral compartment, thereby reducing the load and contact area over the medial compartment. The first references to HTO date back to 1961 (1); for a long time, closing wedge high tibial osteotomy (CWHTO) was the gold standard of treatment in this field. Consequently, there exist numerous studies dealing with aspects (survival and complications) of CWHTO.

CWHTO involves fibular osteotomy, common peroneal nerve dissection, proximal tibiofibular joint disruption, and bone stock loss $(2,3)$. Furthermore, with this procedure it is difficult to obtain gradual correction of the axis. For these reasons, opening wedge high tibial osteotomy (OWHTO) has been gaining popularity in recent years. Moreover, OWHTO allows for better tuning of the osteotomy as well as triplanar and gradual correction. The literature of recent years therefore contains various papers on different aspects of OWHTO: indications, surgical technique, limits and results.

The aim of this study is to review the literature on OWHTO, considering indications and prognostic factors, outcomes and limits of the procedure.

\section{Indications}

Correct patient selection is mandatory for achieving good results with OWHTO $(4,5)$. According to the literature, the factors that could influence the prognosis are: age, body mass index (BMI), grade of OA, range of motion (ROM), and associated instability. In order to gain a better understanding of the correct indications for OWHTO, we have reviewed each of these factors as examined in the most recent literature. 
Age

There is agreement in the literature regarding the association between age and outcomes of HTO. Some Authors found that the risk of failure increased 7.6\% for each year of age (6). At 10 years, the survival rate of HTO in patients younger than 65 years was 90 versus $70 \%$ in patients older than 65 . Recently Howells et al. (7) analyzed 164 patients who underwent HTO, of whom 95 were reviewed at 10 years of follow-up. These Authors found that better survival is associated with a pre-operative Western Ontario and McMaster Universities osteoarthritis index (WOMAC) $>45$ points, age $<55$ years and $\mathrm{BMI}<30$. However, in patients older than 55 years, adequate preoperative functional scores (more than 66.7 on the Japanese Orthopaedic Association Knee Score and a WOMAC greater than 45) (8) might provide good survival and functional outcomes. The Authors recommended the routine use of preoperative functional outcome scores to guide decisionmaking when considering a patient's suitability for HTO (7).

Bonasia et al. (9), studying 99 OWHTOs, found that age was a preoperative variable significantly related to a poor outcome: the risk of unsuccessful surgery was five times higher for patients aged $>56$ years, indicating the possible need to consider a narrower range.

\section{$B M I$}

The relationship between BMI and OWHTO outcomes is still debated in the literature. Flecher et al. (10), studying 313 patients, found that those with a BMI of less than 30 had better outcomes. This finding was confirmed by Howells et al. (7) in their study of 95 HTO patients.

Giagounidis and Sell (11) examined 112 knees (94 patients) after HTO for varus and valgus alignment (the osteotomy was performed on the medial or lateral side in relation to the type of malalignment). They found that patients with a BMI greater than $10 \%$ above normal values had a pain-free period of 5 years, as opposed to 7.8 years for those with a BMI lower than $10 \%$ above normal values (11). Since normal values of BMI range between 18.5 and 24.9, we can say that a BMI greater than 27.4 is associated with poorer outcome. On the other hand, Naudie et al. (12), in a survival analysis of 106 HTOs, found that patients with a BMI lower than 25 have even worse results. On the basis of the hypothesis that lighter patients are often more active, the Authors suggested that this finding could be related to higher stress on the osteotomy site. Bonasia et al. (9), studying 99 OWHTOs, concluded that in patients with a BMI greater than 30 the risk of unsuccessful surgery is 10 times higher, and Akizuki et al. (13) stated that a BMI greater than 27.5 is associated with early failure of the osteotomy. These results are summarized in Table 1. We can conclude that the ideal BMI for HTO is a value of between 25 and 27.5.

\section{Grade of osteoarthritis}

The severity of medial compartment $\mathrm{OA}$ is a relevant

Table 1. BMI and outcomes of OWHTO.

\begin{tabular}{|c|c|c|c|}
\hline Authors (Ref.) & $\mathbf{N}^{\circ}$ of HTOs & Statistical method & Results \\
\hline Howells et al., 2014 (7) & 95 & Kaplan-Meier survivor analysis & $\begin{array}{l}\text { Ten-year survivorship: } 69 \% \text { for BMI }>30 \\
\text { vs } 97 \% \text { for BMI <30 }\end{array}$ \\
\hline Bonasia et al., 2014 (9) & 99 & SLR, MLR & $\begin{array}{l}\text { SLR: BMI }>30 \text { poor WOMAC/ Knee } \\
\text { Society score ( } \mathrm{p} 0.038) \text {; MLR BMI }>30 \text { not } \\
\text { significant ( } \mathrm{p} 0.16 \text { ) }\end{array}$ \\
\hline Akizuki et al., 2008 (13) & 118 & Cox proportional hazard ratio & $\begin{array}{l}\text { BMI }>27.5 \text { early failure, hazard ratio } 0.174 \\
\text { (p } 0.0015)\end{array}$ \\
\hline Giganoudis et al., 1999 (11) & 112 & Kaplan-Meier survivor analysis & $\begin{array}{l}\mathrm{BMI}>\mathrm{NV}+10 \%, \rightarrow \text { pain free period of } \\
5.07 \text { years; } \mathrm{BMI}<\mathrm{NV}+10 \%, \rightarrow \text { pain free } \\
\text { period of } 7.8 \text { years }\end{array}$ \\
\hline
\end{tabular}

$\mathrm{SLR}=$ Simple logistic regression; MLR=multiple logistic regression; $\mathrm{BMI}=$ Body max index; $\mathrm{NV}=$ normal values; OWHTO= open wedge high tibial osteotomy 
predictor of outcome after HTO. In a study by Flecher et al. (10), 23 out of 31 knees (74\%) classified as Ahlback Grade 3 preoperatively required revision at a mean follow-up of 16 years (10). Bonasia et al. found statistically significant better outcomes in patient with Ahlback Grade 0 (9). Floerkemeier (14) evaluated 533 patients at an average of 3.6 years postoperatively; before surgery, $85 \%$ of these patients had shown grade III or IV OA. The Authors reported good outcomes also in patients with severe monocompartmental arthritis, with a $6 \%$ rate of local postoperative complications. Furthermore, they found no correlation between patient age and Oxford Knee Score. These Authors found favorable midterm results in OWHTO even in older patients with a high degree of medial cartilage damage. Despite the observations of Floerkemeier et al., it is generally agreed in the literature that a low degree of arthrosis is linked to better outcomes; on the other hand, it is clear that tricompartimental OA is a contraindication to osteotomy (15, 16). Like Majima et al., we did not find any evidence in the literature that shifting the weight-bearing line towards the lateral compartment worsens the conditions of this compartment (17). In very young patients a neutral alignment seems to be preferable. Correction to a femorotibial angle between $6^{\circ}$ and $14^{\circ}$ of valgus was associated with an optimal clinical result (10). Undercorrection to less than $5^{\circ}$ of femorotibial valgus was associated with a high $(62.5 \%)$ failure rate (18).

\section{Range of motion}

$\mathrm{ROM}$ is another parameter that must be investigated before performing an OWHTO. Different Authors have reported an association between reduced ROM and worse outcomes, with a flexion contracture constituting a contraindication to OWHTO. Berman et al. (19), in a retrospective study on 35 patients undergoing a total of 39 OWHTOs, found that early failure occurred in patients with a $\mathrm{ROM}<90^{\circ}$. Akizuki et al. (13) identified $100^{\circ}$ of preoperative ROM as the threshold value dividing good results from bad ones. A ROM lower than $100^{\circ}$ was linked to a hazard ratio of 6.785 (p-value 0.001), and in the same study the Authors affirmed that a preoperative maximum flexion value lower than $120^{\circ}$ was linked to a hazard ratio of 2.982 (p-value 0.0474) (13). Naudie et al. (12) found that a preoperative ROM lower than $120^{\circ}$ associated with flexion contracture greater than $5^{\circ}$ was related to early failure ( $\mathrm{p}$-value 0.042). Bonasia et al. (9) analyzed 99 OWHTOs; after simple logistic regression, they concluded that a ROM lower than $120^{\circ}$ affected the outcome, producing a fourfold increase in the risk of unsuccessful surgery. However, using a multiple logistic regression model this parameter did not show statistical significance $(p=0.16)$. The same Authors found that a postoperative ROM of less than $120^{\circ}$ significantly affected outcomes, both in multiple and single logistic regression models. In conclusion, as confirmed by other recent studies $(3,4,12,20)$, a preoperative ROM of less than $120^{\circ}$ increases the likelihood of a worse outcome.

\section{Instability}

Combined knee instability and malalignment is a challenging problem for the surgeon. It is important to distinguish between a primary varus, double varus or triple varus knee. As described by Noyes et al. (21), a primary varus is characterized by a primary varus deformity of the lower limb without any ligament instability; this is the best indication for an OWHTO. Double varus is a condition characterized by the presence of a varus bone deformity accompanied by ligament instability, primarily of the anterior cruciate ligament (ACL). Triple varus is characterized by the association of varus deformity with central ligament insufficiency (anterior and posterior cruciate ligaments) and failure of the posterolateral corner. This condition is usually associated with a varus thrust during gait.

Some Authors $(18,22,23)$ reported poor results of HTO in patients with knee joint instability. However, several recent studies seem to argue against knee joint instability as a contraindication to OWHTO. In cases with combined instability and varus malalignment, a combined or staged procedure, including both ligament procedures and OWHTO, can be considered (5, $21,24,25)$. ACL deficiency leads to progression of OA due to increased anterior tibial translation upon weight bearing (25). Noyes et al. (21) demonstrated that in varus angulated ACL-deficient knees, incorrect analysis of the lesion, focusing on ligaments and instability alone and not considering the varus alignment of the knee, leads to failure of the ligamentous reconstruction. These Authors reported on 41 patients. Before HTO was performed, these patients had undergone failed procedures: a total of 72 failed procedures in the group affected by double varus knee 
(23 patients) and 66 in the triple varus knee group (18 patients). These failed surgical interventions were simple arthroscopy, partial or total meniscectomy, meniscus allograft or repair, and lateral collateral or posterolateral reconstruction.

HTO in double and triple varus knees (whether or not it is associated with ligament procedures) gives statistically significant improvements in pain, swelling, giving away, and function during activities of daily living and sport (21).

Because of the difference in shape between the medial and lateral tibia, the medial OWHTO can achieve multiplanar correction. This osteotomy can have effects on the coronal and sagittal plane and, if correctly planned, may help to extend the indications for the procedure, so that they include posterolateral laxity with varus thrust, ACL deficiency and combined ligamentous laxity with varus or posterolateral thrust (5, 26). Savarese et al. (24) reported that the amount of posterior tibial slope after OWHTO depends on the position of the plate used to stabilize the osteotomy. An anteromedial plate increases the slope by an average of $5.5^{\circ}$, while a posteromedial plate tends not to modify the posterior tibial slope (24). Noyes et al. (27) calculated that for each 1-mm increase in the anterior gap, there is a two-degree increase in the posterior tibial slope.

As regards the timing of the procedures in double or triple varus knees, Badhe et al. (25), in their study on 14 patients with knee instability and varus alignment, suggested that those with a double varus knee (varus plus ACL deficiency) do well with single-stage HTO and ACL reconstruction. Triple varus knees (varus knees associated with posterolateral corner and ACL deficiency) do better with an OWHTO than with a CWHTO, as combined osteotomy and ligament reconstruction procedures can be performed without disturbing the proximal tibiofibular joint; if posterolateral structures are lax and not completed disrupted, OWHTO alone tends to stabilize the knee and could avoid ligament reconstruction especially in chronic lesions or if the major symptom is pain and not instability (25). Savarese et al. (24) stated that in patients with a chronic posterior cruciate ligament (PCL)-deficient knee associated with double or triple varus, OWHTO should be performed before soft tissue procedures. Patients should be evaluated 6-8 months later, and if the knee is still unstable, soft tissue reconstruction should be performed. Noyes et al. (21) affirmed that in double varus knees, an arthroscopic gap test of more than $12 \mathrm{~mm}$ at the periphery of the lateral tibiofemoral compartment contraindicates ACL reconstruction at the same time as osteotomy; the same Authors in triple varus knees recommend staging the procedure.

Summarizing the indications for HTO in patients with ligament instability we can say that:

- In the presence of a subacute ACL injury plus a coronal malalignment (varus thrust) or sagittal malalignment (increased tibial slope) the treatment of choice should be an HTO plus ACL reconstruction. It is suggested that this should be done as a one-stage procedure $(28,29)$.

- Chronic ACL injury with varus alignment should be treated with HTO alone or HTO combined with ACL reconstruction, depending on the patient's age, activity level and the reported instability $(28,29)$.

- Chronic ACL plus medial compartment OA or varus thrust should be treated with HTO plus ACL reconstruction as a one-stage procedure or HTO alone, but if there is residual instability this should be followed by delayed ACL reconstruction (twostage procedure).

- If there is a chronic posterolateral instability/laxity, for example in cases of PCL injury or posterior lateral complex injury, possibly associated with an ACL injury, the treatment of choice should be an HTO alone, but if there is residual instability a delayed PCL reconstruction should be done (two-stage procedure) (30).

Table 2 summarizes the best indications for OWHTO according to the literature analyzed.

\section{Planning}

The preoperative planning consists of complete radiographic evaluation of the knee: anteroposterior and lateral long-leg views on weight bearing, associated with the Merchant view of the patella and Rosemberg view of the lower limb $(5,24)$. On the X-rays, the degree of $\mathrm{OA}$, the patellar height (Insall-Salvati, Blackburne-Peel, or Caton-Deschamps index), as well as the lower limb alignment on both the sagittal and the coronal view should be assessed. In patients affected by double and triple varus, panoramic weight-bearing $\mathrm{X}$-rays can show a larger opening in the lateral 
Table 2. Best indications for OWHTO.

\begin{tabular}{lll}
\hline & Indications & Literature \\
\hline Age & $<56$ years & Bonasia et al., 2014 (9) \\
\hline ROM & $>=120$, flexion contracture $<5^{\circ}$ & $\begin{array}{l}\text { Bonasia et al., } 2014 \text { (9); Naudie et al., 1999 (12); Dettoni et } \\
\text { al., 2010 (4) }\end{array}$ \\
\hline BMI & Naudie et al., 1999 (12) Akizuki et al., 2008 (13) \\
\hline Preop. PH & Ahlback I-II & Dettoni et al., 2010 (4) \\
\hline Preop. Scores & CDI $>0.6$ & Portner, 2014 (36) \\
\hline Instability & $\begin{array}{l}\text { Wouble varus }+ \text { acute ACL tear with 1-stage } \\
\text { procedure; triple varus with a two-stage } \\
\text { procedure (OWHTO and, if there is residual } \\
\text { instability at 6-8 months, PCL and posterolateral } \\
\text { corner reconstruction) }\end{array}$ & $\begin{array}{l}\text { Rossi et al., 2011 (5); Noyes et al., 2000 (21); } \\
\text { Savarese et al., 2011 (24) }\end{array}$ \\
\hline
\end{tabular}

$\mathrm{ROM}=$ range of movement; $\mathrm{BMI}=$ body mass index; $\mathrm{ACL}=$ anterior cruciate ligament; OWHTO= open wedge high tibial osteotomy; $\mathrm{PCL}=$ posterior cruciate ligament; $\mathrm{CDI}=$ Caton-Deschamp index; $\mathrm{OA}=$ osteoarthritis; $\mathrm{PH}=$ patellar height.

compartment, compared with the normal side, due to ligament insufficiency of the lateral structures of the knee. Magnetic resonance imaging (MRI) is an important complementary examination that should be requested, as it provides important and detailed information on the degree of cartilage damage. Furthermore, in cases of double and triple varus, MRI helps in the diagnosis of peripheral and central ligament lesions, contributing to the surgical planning (31).

The correction angle is calculated as described by Dugdale et al. (32): from a point representing $3^{\circ}$ to $5^{\circ}$ of mechanical valgus located at $62.5 \%$ of the tibial width (approximately corresponding to the lateral tibial spine), as measured from the tip of the medial edge of the proximal tibia, a single line is drawn to the center of the femoral head. Another line is drawn from this point to the center of the ankle joint. The angle between the two lines represents the angle of correction $(\alpha)$. Next, the osteotomy line is measured from the medial (about $4 \mathrm{~cm}$ below the joint line) to the lateral (tip of the fibular head) side of the proximal tibia. This measurement is transferred to both rays of the $\alpha$ angle from the vertex. In this way, the $\alpha$ angle is defined by two identical segments (a1b1 and a1c) equal to the osteotomy length, which are then connected by another line (b1c). This line serves as the base of an isosceles triangle and corresponds to the opening that should be achieved medially at the osteotomy site (Fig. 1). Dugdale et al. (32) reported that a weight-bearing line crossing the knee lateral to $75 \%$ of the tibial plateau can potentially lead to a lift-off of the medial femoral condyle from the medial tibial pla-

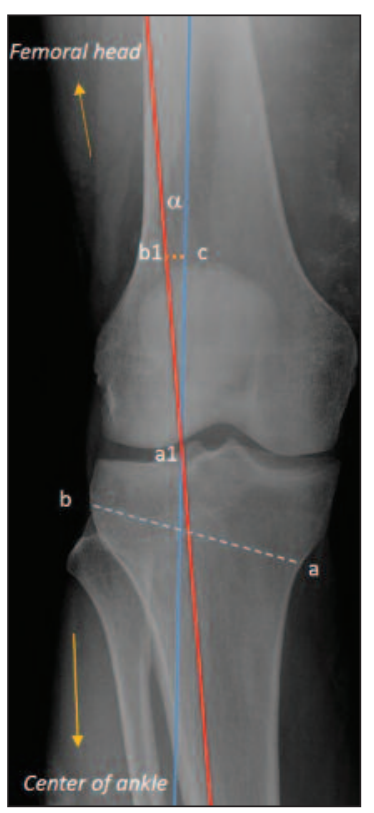
teau. Unicondylar weight bearing resulting from distraction of the medial compartment is undesirable and could result in lateral compartment deterioration. Shifting the weight-bearing line to a

Fig. 1. From a point representing $3^{\circ}$ to $5^{\circ}$ of mechanical valgus located at $62.5 \%$ of the tibial width, a single line is drawn to the center of the femoral head. Another line is drawn from this point to the center of the ankle joint. The center of the ankle joint. The angle between the two lines represents the angle of correction $(\alpha)$. The osteotomy line is measured from the medial (about $4 \mathrm{~cm}$ below the joint line) to the lateral (tip of the fibular head) side of the proximal tibia. This measurement proximal tibia. This measurement is transferred to both rays of the a angle from the vertex. The line, b1c corresponds to the opening that should be achieved medially at the osteotomy site. 
point located at $62.5 \%$ of the tibial plateau allows unloading of the medial compartment and avoids deterioration of the lateral one. According to the affirmations of Noyes et al. (21) and Savarese et al. (24), if $\mathrm{OA}$ of the medial compartment is not present, the mechanical axis should cross a point located at $50 \%$ of the knee.

In patients with double and triple varus, or if OWHTO is performed in association with meniscal transplantation or cartilage procedures (33), the weight-bearing line can be moved to a neutral correction, avoiding unnecessary overcorrection; the aim when calculating the opening wedge correction is to transfer the axis of weight bearing to the center of the knee, i.e. to achieve a final mechanical angle of $0^{\circ}(21)$. This is done in the same way as in cases of primary varus, but the line from the center of the ankle and from the head of the femur cross in the center of the knee, forming the angle of correction.

\section{Surgical technique: OWHTO}

The patient is positioned supine on a radiolucent operating table, and a tourniquet is placed around the thigh. Arthroscopy should be performed first to manage or assess associated pathologies. A longitudinal incision is made starting from $1 \mathrm{~cm}$ below the joint line and midway between the medial border of the tibial tubercle and the posteromedial border of the tibia. The pes anserinus is retracted distally with a blunt retractor, exposing the superficial medial collateral ligament. The ligament is partially detached distally with a Cobb elevator, avoiding complete distal insertion detachment, and the medial border of the patellar tendon is located and retracted. A guidewire is inserted, under fluoroscopic control, beginning at the anteromedial tibia at the level of the superior border of the tibial tubercle, approximately $4 \mathrm{~cm}$ distal to the joint line, to avoid damaging the lateral cortex. The guidewire direction is towards the tip of the fibular head $1 \mathrm{~cm}$ below the lateral articular surface. The osteotomy is performed immediately distally to the guidewire, using an oscillating saw for the medial cortex. The osteotomy is then completed within $1 \mathrm{~cm}$ of the lateral tibial cortex using graduated thin flexible osteotomes. Two or three osteotomes can be piled up at the osteotomy site to achieve the desired opening. Calibrated wedges are then inserted into the osteotomy in order to achieve the planned degree of correction. The accuracy of the correction is verified using a long alignment rod under fluoroscopic control. The rod should lie at approximately $62.5 \%$ of the tibial width (just lateral to the lateral tibial spine). The opening of the osteotomy obtained is synthesized with the chosen plate and two cancellous screws proximally and two cortical screws distally (Fig. 2). If the opening measures more than $10 \mathrm{~mm}$ bone grafting is required (5).

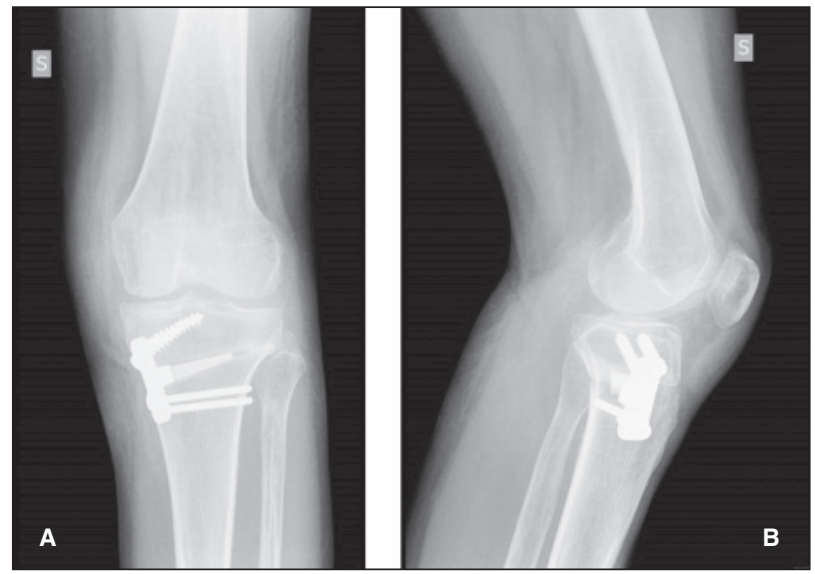

Fig. 2. Postoperative X-rays at 3 months: A: AP view of the operated knee; B: lateral view of the operated knee.

\section{Limits}

The literature on OWHTO describes three main limits of the procedure: the risk of increasing the tibial slope, the risk of a lowering of the patella, and the possible difficulty converting to total knee arthroplasty (TKA) (20, 34-36).

\section{Modification of the tibial slope}

It is universally accepted that HTO can modify the tibial slope, but there is still some disagreement over the extent and, therefore, severity of this modification. The variability in the literature might be explained by the different methods used to calculate the slope $(27,37)$.

Martin et al. (35) analyzed 323 consecutive OWHTO procedures. They divided the possible complications occurring after HTO into three classes: 1. adverse 


\section{p oints}

M. Corgiat Loia et al.

events not necessitating further treatments; 2. adverse events requiring additional or extended non-operative management; 3. adverse events necessitating reoperation or long-term medical care. A tibial slope increase of more than $10^{\circ}$ was found in just $1 \%$ of cases and was classified as a class 1 complication. The Authors concluded that tibial slope increase does not seem to influence the WOMAC score.

The tibial slope variations in OWHTO and CWHTO demonstrated by Ducat et al. (34) were smaller than those usually described in the literature (changes of less than $1^{\circ}$ ). These Authors concluded that, when the surgical technique was respected, OWHTO resulted in very few side effects in terms of tibial slope changes. Because of the configuration of the tibia, an OWHTO in which the anterior osteotomy gap is equal to the posterior one will increase the slope, decrease knee extension and potentially increase ACL tensile load. On the other hand, a CWHTO with equally sized anterior and posterior gaps will have little effect on the slope. Noyes et al. (27) developed a trigonometric calculation method according to which the vertical gap measurements of the opening wedge osteotomy (A1, A2) are functions of the distance from the hinge axis of the lateral tibial cortex of the opening wedge (B1, $\mathrm{B} 2)$, the oblique angle of the anteromedial tibial cortex $\alpha$, and the distance along the osteotomy site (L) (Fig. 3). Each millimeter of difference in the anterior gap may increase the tibial slope by approximately $2^{\circ}$. To maintain the normal tibial slope, the height of the osteotomy at the posteromedial cortex will always be greater than at the tibial tubercle. Slope modification can be controlled by using the correct technique and ensuring correct planning. Furthermore, as highlighted in the literature, slope modification can be useful in the treatment of knee instabilities and should be considered a true limit of OWHTO $(24,28)$.

\section{Patellar height}

The risk of obtaining a patella baja after OWHTO is reported in the literature, but the issue of how to avoid this is still debated. Longino et al. (38) affirmed that combining tibial tubercle osteotomy with OWHTO decreases the risk of patella baja.

Oliver et al. suggested that the preoperative height of the patella may determine the choice of HTO technique (CWHTO, OWHTO or combined technique). In their study, they demonstrated that closing wedge

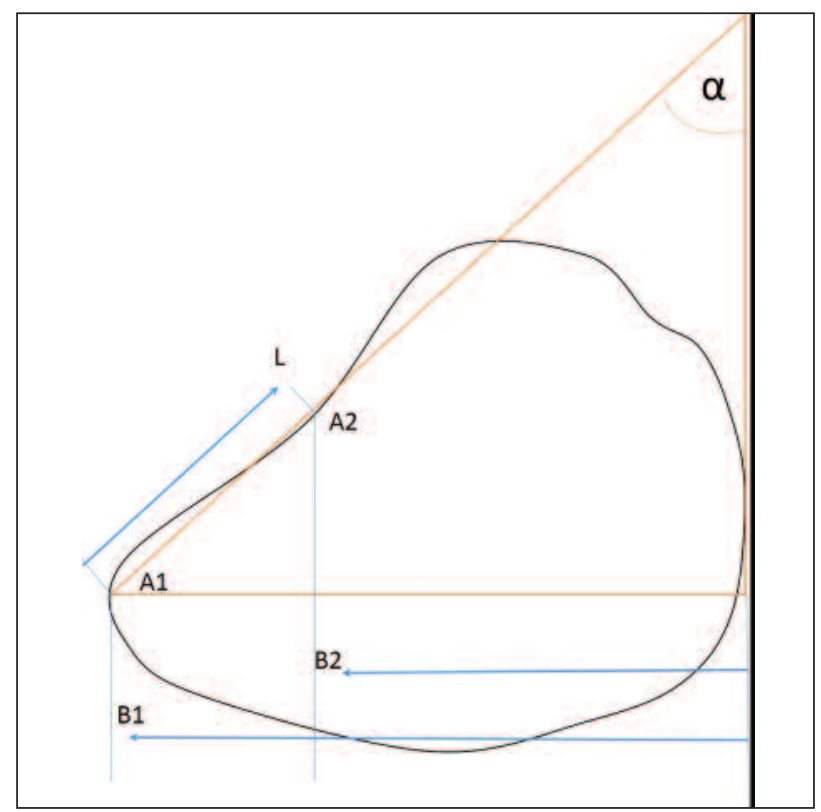

Fig. 3. Vertical gap measurements are functions of the distance from the hinge axis of the lateral tibial cortex (B1, B2), the oblique $\alpha$ angle of the anteromedial tibial cortex, and the distance along the osteotomy $L$. These functions are $L=(B 1-B 2) \operatorname{COS}(\alpha)$; B2 $=[(A 2)(B 1)] / A 1$.

osteotomy produced patellar ascent in $13 \%$ of cases; patella baja was detected in $21 \%$ of patients undergoing opening wedge osteotomy, while the change in patellar height was minimal with combined osteotomy. Chae et al. (39) found a decrease in the distance between the patella and tibiofemoral joint line in $82 \%$ of 32 patients submitted OWHTO. $26 \%$ of postoperative Blackburne-Peel values satisfied the radiographic criterion for patellar infera (Blackburne-Peel ratio $<0.54)$. It has been reported that the decreased distance between the patella and the tibiofemoral joint line following medial OWHTO is a function of joint line elevation, and that the high incidence of patella infera following medial OWHTO may have deleterious effects on patellofemoral biomechanics or may complicate subsequent total knee arthroplasty (40).

Summarizing what we found in the literature, changes in patellar height may be due to three factors: joint line elevation with respect to the anterior tibial tubercle, which occurs when OWHTO is carried out in the supra-tuberosity metaphysis; tibial slope modification; patellar ligament relaxation or shortening for whatever reason (quadriceps collapse or late dystrophic phenomena). El 
Amrani et al. (41), in their study of 40 OWHTOs, observed that a mean $1.5^{\circ}$ increase in tibial slope tended to be associated with decreased patellar height, but this correlation failed to achieve significance. Joint line elevation can be controlled through adequate preoperative planning. El Amrani et al. (41) found no correlation between degree of osteotomy opening and patellar height loss with any index used.

Patellar height modifications due to ligament relaxation or shortening have been described by a number of Authors (41-43); all agreed that rigid internal fixation with early rehabilitation could reduce the incidence of these modifications. El Amrani et al. (41) suggested not using OWHTO in the presence of a preoperative Caton-Deschamps index of around 0.6. Portner (36), in his study of 76 osteotomies of different types (OWHTO, CWHTO or combined), suggested that the preoperative patellar height should determine which type of osteotomy is chosen. He recommended that surgeons avoid lateral closing wedge osteotomies when the patella is already very high (plateau-patella angle greater than $29^{\circ}$ ), and that they avoid opening wedge osteotomies when the patella is lower than the normal mean of $25^{\circ}$. Savarese et al. (24) suggested that if the anterior gap of the osteotomy is greater than $1 \mathrm{~cm}$, it is better to perform an osteotomy to lift the tibial tubercle by the same amount, in order to avoid patella baja.

\section{Conversion to total knee arthroplasty (TKA)}

TKA after HTO is a technically demanding procedure, and there is concern that a previous HTO can jeopardize the outcome of a TKA.

The literature studies reporting worse outcomes of TKA after HTO compared with primary TKA do not distinguish between OWHTO and CWHTO. Haslam et al. (44) compared 51 TKAs after HTO (the Authors considered closing and opening wedge osteotomies together) with a matched group of primary TKAs. They found that although the overall Hospital for Special Surgery scores showed no significant difference between the two groups, more patients in the osteotomy group showed poor results $(\mathrm{p}=0.027)$, significantly reduced flexion $(\mathrm{p}=0.006)$, and a higher rate of reoperation.

When unicompartmental knee arthroplasty (UKA), OWHTO and CWHTO are considered separately the results are different. Niinimaki et al. (45) in their huge study (1,036 patients with previous HTO compared with 4,143 patients who underwent primary TKA) reported that despite the increased technical difficulties encountered in TKA after HTO, revision rates were only slightly higher. On the contrary, Robertsson et al. (46) found a significantly higher failure rate for TKAs performed after previous CWHTOs $(n=356)$ or UKAs $(\mathrm{n}=920)$ than for primary TKAs $(\mathrm{n}=118,229$ patients) (risk ratio, 2.8; confidence interval [C] , 2.23.5; $\mathrm{p}<0.001$, and $1.7 \mathrm{CI}, 1.1-2.6$; $\mathrm{p}<0.001$, respectively). However, the same Authors, comparing 482 OWHTOs with the 118,229 de novo TKAs, found no statistically significant differences. Moreover, in this study, stemmed implants were used in $17 \%$ of the revised UKAs and in just 4\% of the revised HTOs. Bastos Filho et al. (47) found an increased need for tibial tubercle osteotomy in TKA after CWHTO and for medial release in patients previously submitted to OWHTO.

\section{Other debated issues}

\section{Stabilization of the osteotomy}

Failure of the plate used for the fixation is one of the class 3 complications described by Martin et al. (35) and it requires reoperation. In their finite element analysis, Golovakha et al. (48) concluded that different fixation devices generate different fixation stability profiles for different opening wedge sizes. On the basis of computational simulations, none of the studied fixation types warranted intransigent full weight bearing. The highest fixation stability was observed for the TomoFix plates (Depuy Synthes; Oberdorf, Switzerland) and the lowest for the first generation of Puddu plate (Arthrex; Naples, Florida).

Hernigou et al. (49) reported good results using Limmed locked plate fixation (Limmed; Pontoise, France). They reported that all the 50 patients who had undergone OWHTO synthesized with this tool were allowed immediate weight bearing. These patients were compared with a further 50 patients treated with other devices and without immediate weight bearing. Statistically significant differences were seen between the groups in terms of both radiographic union and radiographic stability. The patients in the 
Limmed group showed a shorter time for union (average four weeks difference) without loss of correction during healing. On radiographic evaluation, there was a significant increase in osseointegration in the group with weight bearing compared with the control group. Computed tomography scans of the grafted area at four months after surgery showed no significant difference between the two groups in the quality of the newly formed bone.

We prefer to use the second generation of Puddu plates with two cancellous screws proximal to the osteotomy and two cortical screws distal to the osteotomy. This construct does not allow immediate weight bearing after surgery, but if the medial hinge is preserved, it has proven its efficacy. This device allows a smaller incision than other plates and has a simple instrumentation allowing a shorter intervention time (for example, a Tomofix plate has four screws on the proximal side of the osteotomy and four in the distal part of the plate). The shape of the plate with a wedge that supports the osteotomy allows better tuning of the osteotomy and better positioning of the plate.

\section{UKA vs HTO}

In the literature there is debate over whether UKA could be an alternative to HTO in the treatment of unicompartmental $\mathrm{OA}$ in young patients affected by varus knee. Some Authors have reported that UKA led to better outcomes with lower complication rates (50), while others found that the results obtained with UKA were superior only at a short-term follow-up (51). Akizuki et al. (13), in their prospective study of 159 knees, achieved good results with HTO in $73.7 \%$. In our opinion these studies are not representative of the true outcomes of OWHTO because there is a very limited overlap of indications between UKA and OWHTO, and also because in these studies the osteotomy chosen was often a closing wedge osteotomy and not an opening wedge one.

As described by Dettoni et al. (4), the only patients who fit both indications (UKA and OWHTO) are moderately active men and women aged between 55 and 65 years, with a preoperative alignment of $5-10^{\circ}$ of varus, a $\mathrm{ROM}$ of $100^{\circ}$, less than $5^{\circ}$ of flexion contracture, and Grade II (Ahlback scale) medial compartment OA. Respecting the correct indications for OWHTO, Dettoni et al. (4) concluded that OWHTO outcomes are similar to those of UKA. OWHTO showed a slightly better ROM and a somewhat lower risk of revision in the first years of follow-up; these Authors suggested that the advantages of OWHTO over UKA are the ability to maintain a higher level of activity without potential wear of arthroplasty components. For these reasons, Richmond (52) affirmed that HTO seems to be a better choice than UKA for younger and more active patients. The advantages of UKA are the possibility of replacing a severely damaged compartment (any Ahlback grade), the preservation of bone stock, the faster recovery time and the minimal invasiveness in comparison with TKA; these features make this treatment the ideal choice in older patients ( $>65$ years old). In the study by Lobenhoffer et al. in 533 patients fulfilling the abovementioned criteria, the subjective ratings of OWHTO were better than in comparable groups treated with UKA and with TKA (53).

Yim et al. failed to identify any significant differences between HTO and UKA for medial unicompartmental OA in terms of return to recreational activity and short-term clinical outcomes (54).

We conclude that the indications for UKA and HTO do not totally overlap and that, respecting the correct indications, good results can be obtained with both the treatments.

\section{Results of HTO}

The literature lacks studies describing long-term outcome of HTO, and few of those that do exist concern OWHTO. Indeed, most of the studies with a longer follow-up deal with CWHTO since the technique has a longer history (and, therefore, a larger number of documented cases). There is still no agreement on which is the best osteotomy in terms of outcome. Soleimanpour et al. (55), in their study of 42 knees, found statistically significant differences (in operation duration, weight bearing duration and return to routine activities) between OWHTO and CWHTO, in favor of OWHTO. Harris et al. (51), in a systematic review analyzing 69 studies (4,557 subjects), evaluated survivorship and outcomes of isolated HTO and HTO combined with other procedures. They concluded that at two years of follow-up the survivorship was statisti- 
Table 3. Outcomes of OWHTO in literature.

\begin{tabular}{|c|c|c|c|c|c|}
\hline Author & $\begin{array}{l}\mathrm{N}^{\circ} \text { cases } \\
\text { (mean age) }\end{array}$ & Technique & Implant & Follow-up & Outcomes \\
\hline $\begin{array}{l}\text { (Martin et al., } \\
2014 \text { (35) }\end{array}$ & 323 (46.3 y) & OWHTO & $\begin{array}{l}\text { Tomofix } \\
{ }^{\circledR} \\
\text { and Puddu }^{\circledR}\end{array}$ & $2 y$ & $\begin{array}{l}\text { WOMAC: class } 1 \text { ( } 76 \text { at } 6 \text { months; } 80 \text { at } 12 \text { months; } \\
80 \text { at } 24 \text { months); Class } 2 \text { ( } 76 \text { at } 6 \text { months, } 80 \text { at } 12 \\
\text { months, } 76 \text { at } 24 \text { months); Class } 3 \text { ( } 56 \text { at } 6 \text { months, } 74 \\
\text { at } 12 \text { months, } 82 \text { at } 24 \text { months) }\end{array}$ \\
\hline
\end{tabular}

\begin{tabular}{|c|c|c|c|c|c|}
\hline $\begin{array}{l}\text { Floerkemeier et al., } \\
2013 \text { (14) }\end{array}$ & 386 & OWHTO & Tomofix $^{\circledR}$ & $3.6 \mathrm{y}$ & Mean Oxford Knee score 43 (range 8-48) \\
\hline Saito et al., 2014 (63) & $78(68 \mathrm{y})$ & OWHTO & - & $6.5 \mathrm{y}$ & $\begin{array}{l}\text { Knee Society Score from } 49.6 \text { (SD } 11.4,26 \text { to } 72 \text { ) } \\
\text { preoperatively to } 88.1 \text { (SD } 12.5,14 \text { to } 100 \text { ) }\end{array}$ \\
\hline $\begin{array}{l}\text { Saragaglia et al., } \\
2014 \text { (64) }\end{array}$ & $83(50.4 \mathrm{y})$ & $\begin{array}{l}62 \text { OWHTO; } \\
21 \text { double level } \\
\text { osteotomies }\end{array}$ & - & $5.75 \mathrm{y}$ & $\begin{array}{l}85.5 \% \text { of patients resumed sports activities, } 79.5 \% \text { at } \\
\text { the same level as before surgery. Lysholm score increa- } \\
\text { sed from } 62.5 \pm 15.53 \text { points to } 90.49 \pm 8.62 \text { points. } \\
\text { Tegner score and UCLA score did not decrease }\end{array}$ \\
\hline $\begin{array}{l}\text { Duivenvoorden } \\
\text { et al., } 2014 \text { (57) }\end{array}$ & 92 (49.9y) & $\begin{array}{l}47 \text { CWHTO } \\
45 \text { OWHTO }\end{array}$ & Puddu ${ }^{\circledR}$ & $7.3 \mathrm{y}$ & $\begin{array}{l}\text { HSS score for OWHTO increased one year after } \\
\text { surgery (baseline } 72.3 \pm 9.5 \text {; one year } 80.9 \pm 13.5 \text {; six } \\
\text { years } 80.8 \pm 13.8 \text { ) }\end{array}$ \\
\hline
\end{tabular}

\begin{tabular}{|c|c|c|c|c|c|}
\hline $\begin{array}{l}\text { Niemeyer et al., } \\
2010(65)\end{array}$ & $69(47 y)$ & OWHTO & Tomofix $^{\circledR}$ & $3 y$ & $\begin{array}{l}\text { IKDC score increased from } 47.2 \pm 18.71 \text { to } \\
72.7 \pm 17.15 \text {. Lysholm score increased from } \\
54.2 \pm 20.76 \text { to } 79.1 \pm 16.63\end{array}$ \\
\hline
\end{tabular}

\begin{tabular}{|c|c|c|c|c|c|}
\hline $\begin{array}{l}\text { Harris et al., } \\
2013 \text { (51) }\end{array}$ & $\begin{array}{l}4344 \\
(69 \text { meta- } \\
\text { analysis } \\
\text { studies) }\end{array}$ & $\begin{array}{l}1625 \\
\text { OWHTO } \\
2486 \\
\text { CWHTO }\end{array}$ & - & $7.1 \mathrm{y}$ & $\begin{array}{l}\text { HSS increased from } 71.2 \pm 9.7 \text { to } 91.3 \pm 6.4 \text { at } 7.5 \text { years } \\
\text { Lysholm score increased from } 60.5 \pm 14 \text { to } 83 \pm 13 \text { at } \\
7.5 \text { years. MCS from } 44.6 \pm 2.4 \text { to } 70.7 \pm 7.8 \text { at } 3 \text { years. } \\
\text { SF36 MCS from } 75.8 \pm 15 \text { to } 83.0 \pm 15 \text { at } 2 \text { years }\end{array}$ \\
\hline
\end{tabular}

\begin{tabular}{llllll}
\hline $\begin{array}{l}\text { Hernigou et al., } \\
2010 \text { (66) }\end{array}$ & $53(60 \mathrm{y})$ & $\begin{array}{l}\text { OWHTO }+ \\
\text { beta-TCP }\end{array}$ & $\begin{array}{l}\text { Buttress } \\
\text { plate }\end{array}$ & $10 \mathrm{y}$ & $\begin{array}{l}\text { Preop scores HHS no pain in 40, slight pain in 2; } \\
\text { moderate/severe pain in 6; severe pain in 6. }\end{array}$ \\
$\begin{array}{l}\text { DeMeo et al., } \\
2010 \text { (60) }\end{array}$ & $20(49 \mathrm{y})$ & OWHTO & Puddu $^{\circledR}$ & $8 \mathrm{y}$ & $\begin{array}{l}\text { Preop. scores: HHS 75.9 (range, 60-91); Lysholm } \\
\end{array}$ \\
& & & & $\begin{array}{l}54.2 \text { (range, 34-67); Postop scores: HHS 86.8 (range, } \\
70-100), \text { Lysholm 83 (range, 70-100) }\end{array}$
\end{tabular}

\begin{tabular}{|c|c|c|c|c|c|}
\hline $\begin{array}{l}\text { Schröter et al., } \\
2011(67)\end{array}$ & $71(40 \mathrm{y})$ & OWHTO & HTO plate & $1 \mathrm{y}$ & $\begin{array}{l}\text { Preop scores: Lysholm } 55.5 \pm 21.7 \text {; HHS } 74.8 \pm 11.7 \text {; } \\
\text { Tegner } 2.6 \pm 0.9 \text {; IKDC } 43 \pm 14.9 \text {. Postop ( } 1 \text { y) scores: } \\
\text { Lysholm } 73 \pm 23.9 \text {; HHS } 87.8 \pm 11.0 \text {; Tegner } 3.7 \pm 1.8 \text {; } \\
\text { IKDC } 66.1 \pm 21\end{array}$ \\
\hline $\begin{array}{l}\text { Laprade et al., } \\
2012 \text { (68) }\end{array}$ & $47(40.5 \mathrm{y})$ & OWHTO & Puddu ${ }^{\circledR}$ & $3.6 \mathrm{y}$ & $\begin{array}{l}\text { MCS. Preop } 42.9 \text { (range, } 8 \text { to 63) Postop } 65.1 \text { (range, } \\
10 \text { to } 100 \text { ) }\end{array}$ \\
\hline $\begin{array}{l}\text { (Bode et al., } \\
2015 \text { (59) }\end{array}$ & $51(47 y)$ & OWHTO & Tomofix $^{\circledR}$ & $5 \mathrm{y}$ & $\begin{array}{l}\text { Preop scores: IKDC } 44.6 \pm 17.8 \text {, Lysholm } 52.1 \pm 20.8 \text {; } \\
\text { Postop }(5 \text { y) scores: IKDC } 69.4 \pm 18.6 ; \text { Lysholm } \\
76.6 \pm 20.5\end{array}$ \\
\hline $\begin{array}{l}\text { Bonasia et al., } \\
2014(9)\end{array}$ & 99 (54 y) & $\begin{array}{l}\text { OWHTO } \\
\text { (1 autologous } \\
\text { iliac bone graft } \\
+60 \text { HA-TCP } \\
\text { substitute })\end{array}$ & Puddu $\AA$ & $7.5 \mathrm{y}$ & $\begin{array}{l}\text { KSS increased from } 135.6 \pm 33.9 \text { to } 160.5 \pm 26.3 \text { at } 5 \\
\text { years. WOMAC increased from } 50.7 \pm 20.8 \text { to } \\
76.1 \pm 18.5 \text { at } 5 \text { years }\end{array}$ \\
\hline
\end{tabular}

OWHTO=open wedge high tibial osteotomy; CWHTO=close wedge high tibial osteotomy; WOMAC= Western Ontario and McMaster Universities Osteoarthritis Index; UCLA=University of California Los Angeles; HSS= Hospital for Special Surgery score; IKDC=International Knee Documentation Committee score; MCS=Modified Cincinnati score; TCP=tricalcium phosphate; HA=hydroxyapatite. 
cally better with OWHTO than with CWHTO. Deie et al. (56) asserted that OWHTO reduced varus moment and lateral thrust, whereas CWHTO had little effect in terms of reducing lateral thrust. Other authors, such as Duivenvoorden et al. (57) and Brouwer et al. (58), did not find any differences.

Table 3, in which we report the most important studies in the literature describing the results of OWHTO, shows that follow-up of these procedures is limited: the longest follow-up we are able to report for OWHTO, found in a meta-analysis, is 20 years (51). In this study the survivorship of the opening wedge osteotomy at 5, 10, 15 and 20 years was 92.4, $84.5,77.3$ and $72.3 \%$, respectively.

Floerkeimeier et al. (14) reported that no osteotomy failed at 3.6 years. Bode et al. (59), in their study of 62 patients who underwent OWHTO, reported a survivorship of $96 \%$ at five years. DeMeo (60) reported an eight-year survival rate of $70 \%$ (14 out of a total of 20 OWHTOs).

Bonasia et al. (9) reported a survivorship of $98.5 \%$ at five years and $75.9 \%$ at 7.5 years. This is the largest study we found in literature with a midterm follow-up. The good outcomes reported and the absence of loss of correction after the osteotomy must be attributed to accurate patient selection, respecting the abovementioned criteria, as well as to a cautious postoperative regimen including early mobilization and adequate rehabilitation, preventing postoperative ROM reduction. This is important as a postoperative ROM of less than $120^{\circ}$ is a unfavorable prognostic factor, as shown by Bonasia et al. (9).

Midterm outcomes of OWHTO reported in the literature are good, characterized by improvements in functional scores. Fashingbauer et al. (61) reported a $92.3 \%$ rate of return to pre-operative sports activities after surgery, with a shift to lower impact activities. In the opinion of the Authors, HTO allows the young, active patient with medial $\mathrm{OA}$ of the knee to return to work, at the same intensity as before, and also to return to sport.

Bonnin et al. (62) observed that among patients under 75 years of age at 50 months of follow-up, $28 \%$ regularly participated in strenuous sports, but $40 \%$ were motivated for these activities. Sixty six percent of the motivated patients regularly participated in at least one impact sport (62).

\section{Conclusions}

On the basis of what we found in literature, the ideal patient for an OWHTO is young (under 56 years of age), is of normal weight or only slightly overweight (BMI 25-27.5), has a range of motion of at least $120^{\circ}$, a flexion contracture of less than $5^{\circ}$, a low grade of medial compartment OA $(<$ Ahlback II) without involvement of lateral compartment or patellofemoral compartment, and has a Caton-Deschamps index > 0.6 and a WOMAC score $>45$ points. The literature shows that regardless of whether or not the procedure is staged, if it is correctly planned instability is not a real contraindication for OWHTO $(5,21,24)$. When this indication was respected and the limitations of the procedure were taken into account, OWHTO showed good outcomes in the literature, with a mean 5year and 10-year survivorship of $89.3 \%$ (range 75 $98.7 \%$ ) and $77.6 \%$ (range 51-97.6\%), respectively (20). Longer follow-up studies regarding OWHTO are certainly required.

\section{References}

1. Jackson JP, Waugh W. Tibial osteotomy for osteoarthritis of the knee. J Bone Joint Surg Br. 1961;43-B:746-51.

2. Preston S, Howard J, Naudie D, et al. Total knee arthroplasty after high tibial osteotomy: no differences between medial and lateral osteotomy approaches. Clin Orthop Relat Res. 2014;472:105-110.

3. Bonasia DE, Governale G, Spolaore S, et al. High tibial osteotomy. Curr Rev Musculoskelet Med. 2014;7:292-301.

4. Dettoni F, Bonasia DE, Castoldi F, et al. High tibial osteotomy versus unicompartmental knee arthroplasty for medial compartment arthrosis of the knee: a review of the literature. Iowa Orthop J. 2010;30:131-140.

5. Rossi R, Bonasia DE, Amendola A. The role of high tibial osteotomy in the varus knee. J Am Acad Orthop Surg. 2011;19:590-599.

6. Trieb K, Grohs J, Hanslik-Schnabel B, et al. Age predicts outcome of high-tibial osteotomy. Knee Surg Sports Traumatol Arthrosc. 2006;14:149-152.

7. Howells NR, Salmon L, Waller A, et al. The outcome at ten years of lateral closing-wedge high tibial osteotomy: determinants of survival and functional outcome. Bone Joint J. 2014;96-B:1491-1497.

8. Goshima K, Sawaguchi T, Sakagoshi D, et al. Age does not affect the clinical and radiological outcomes after openwedge high tibial osteotomy. Knee Surg Sports Traumatol Arthrosc. 2015 Nov 3. [Epub ahead of print]

9. Bonasia DE, Dettoni F, Sito G, et al. Medial opening wedge high tibial osteotomy for medial compartment overload/ arthritis in the varus knee: prognostic factors. Am J Sports Med. 2014;42:690-698. 
10. Flecher X, Parratte S, Aubaniac JM, et al. A 12-28-year follow up study of closing wedge high tibial osteotomy. Clin Orthop Relat Res. 2006;452:91-6.

11. Giagounidis EM, Sell S. High tibial osteotomy: factors influencing the duration of satisfactory function. Arch Orthop Trauma Surg. 1999;119:445-449.

12. Naudie D, Bourne RB, Rorabeck $\mathrm{CH}$, et al. The Install Award. Survivorship of the high tibial valgus osteotomy. A 10- to -22-year follow-up study. Clin Orthop Relat Res. 1999(367):18-27.

13. Akizuki S, Shibakawa A, Takizawa T, et al. The long-term outcome of high tibial osteotomy: a ten- to 20-year followup. J Bone Joint Surg Br. 2008;90:592-596.

14. Floerkemeier S, Staubli AE, Schroeter S, et al. Outcome after high tibial open-wedge osteotomy: a retrospective evaluation of 533 patients. Knee Surg Sports Traumatol Arthrosc. 2013;21:170-180.

15. Aglietti P, Rinonapoli E, Stringa G, et al. Tibial osteotomy for the varus osteoarthritic knee. Clin Orthop Relat Res. 1983;(176):239-251.

16. Ivarsson I, Myrnerts R, Gillquist J. High tibial osteotomy for medial osteoarthritis of the knee. A 5 to 7 and 11 year followup. J Bone Joint Surg Br. 1990;72:238-244.

17. Majima T, Yasuda K, Katsuragi R, et al. Progression of joint arthrosis 10 to 15 years after high tibial osteotomy. Clin Orthop Relat Res. 2000;(381):177-184.

18. Rudan JF, Simurda MA. High tibial osteotomy. A prospective clinical and roentgenographic review. Clin Orthop Relat Res. 1990;(255):251-256.

19. Berman AT, Bosacco SJ, Kirshner S, et al. Factors influencing long-term results in high tibial osteotomy. Clin Orthop Relat Res. 1991;(272):192-198.

20. Amendola A, Bonasia DE. Results of high tibial osteotomy: review of the literature. Int Orthop. 2010;34:155-160.

21. Noyes FR, Barber-Westin SD, Hewett TE. High tibial osteotomy and ligament reconstruction for varus angulated anterior cruciate ligament-deficient knees. Am J Sports Med. 2000;28:282-296.

22. Stukenborg-Colsman C, Wirth CJ, Lazovic D, et al. High tibial osteotomy versus unicompartmental joint replacement in unicompartmental knee joint osteoarthritis: 7-10-year follow-up prospective randomised study. Knee. 2001;8:187-194.

23. Segal NA, Buckwalter JA, Amendola A. Other surgical techniques for osteoarthritis. Best Pract Res Clin Rheumatol. 2006;20:155-176.

24. Savarese E, Bisicchia S, Romeo R, et al. Role of high tibial osteotomy in chronic injuries of posterior cruciate ligament and posterolateral corner. J Orthop Traumatol. 2011;12:1-17.

25. Badhe NP, Forster IW. High tibial osteotomy in knee instability: the rationale of treatment and early results. Knee Surg Sports Traumatol Arthrosc. 2002;10:38-43.

26. Amendola A. The role of osteotomy in the multiple ligament injured knee. Arthroscopy. 2003;19 Suppl 1:11-13.

27. Noyes FR, Goebel SX, West J. Opening wedge tibial osteotomy: the 3-triangle method to correct axial alignment and tibial slope. Am J Sports Med. 2005;33:378-387.

28. Naudie DD, Amendola A, Fowler PJ. Opening wedge high tibial osteotomy for symptomatic hyperextension-varus thrust. Am J Sports Med. 2004;32:60-70.

29. Phisitkul P, Wolf BR, Amendola A. Role of high tibial and distal femoral osteotomies in the treatment of lateral-posterolateral and medial instabilities of the knee. Sports Med Arthrosc. 2006;14:96-104.

30. Arthur A, LaPrade RF, Agel J. Proximal tibial opening wedge osteotomy as the initial treatment for chronic posterolateral corner deficiency in the varus knee: a prospective clinical study. Am J Sports Med. 2007;35:1844-1850.

31. Murphy SB. Tibial osteotomy for genu varum. Indications, preoperative planning, and technique. Orthop Clin North Am. 1994;25:477-482.

32. Dugdale TW, Noyes FR, Styer D. Preoperative planning for high tibial osteotomy. The effect of lateral tibiofemoral separation and tibiofemoral length. Clin Orthop Relat Res. 1992;(274):248-264.

33. Bonasia DE, Amendola A. Combined medial meniscal transplantation and high tibial osteotomy. Knee Surg Sports Traumatol Arthrosc. 2010;18:870-873.

34. Ducat A, Sariali E, Lebel B, et al. Posterior tibial slope changes after opening- and closing-wedge high tibial osteotomy: a comparative prospective multicenter study. Orthop Traumatol Surg Res. 2012;98:68-74.

35. Martin R, Birmingham TB, Willits $\mathrm{K}$, et al. Adverse event rates and classifications in medial opening wedge high tibial osteotomy. Am J Sports Med. 2014;42:1118-1126.

36. Portner O. High tibial valgus osteotomy: closing, opening or combined? Patellar height as a determining factor. Clin Orthop Relat Res. 2014;472:3432-3440.

37. Akamatsu Y, Sotozawa M, Kobayashi H, et al. Usefulness of long tibial axis to measure medial tibial slope for opening wedge high tibial osteotomy. Knee Surg Sports Traumatol Arthrosc. 2014 Oct 29 [Epub ahead of print].

38. Longino PD, Birmingham TB, Schultz WJ, et al. Combined tibial tubercle osteotomy with medial opening wedge high tibial osteotomy minimizes changes in patellar height: a prospective cohort study with historical controls. Am J Sports Med. 2013;41:2849-2857.

39. Chae DJ, Shetty GM, Lee DB, et al. Tibial slope and patellar height after opening wedge high tibia osteotomy using autologous tricortical iliac bone graft. Knee. 2008;15:128-133.

40. Wright JM, Heavrin B, Begg M, et al. Observations on patellar height following opening wedge proximal tibial osteotomy. Am J Knee Surg. 2001;14:163-173.

41. El Amrani MH, Lévy B, Scharycki S, et al. Patellar height relevance in opening-wedge high tibial osteotomy. Orthop Traumatol Surg Res. 2010;96:37-43.

42. Dohin B, Migaud H, Gougeon F, et al. Effect of a valgization osteotomy with external wedge removal on patellar height and femoro-patellar arthritis. Acta Orthop Belg. 1993;59:6975 .

43. Kaper BP, Bourne RB, Rorabeck $\mathrm{CH}$, et al. Patellar infera after high tibial osteotomy. J Arthroplasty. 2001;16:168-173.

44. Haslam P, Armstrong M, Geutjens G, et al. Total knee arthroplasty after failed high tibial osteotomy long-term followup of matched groups. J Arthroplasty. 2007;22:245-250.

45. Niinimaki T, Eskelinen A, Ohtonen P, et al. Total knee arthroplasty after high tibial osteotomy: a registry-based casecontrol study of 1,036 knees. Arch Orthop Trauma Surg. 2014;134:73-77.

46. Robertsson O, W-Dahl A. The risk of revision after TKA is affected by previous HTO or UKA. Clin Orthop Relat Res. 2015;473:90-93.

47. Bastos Filho R, Magnussen RA, Duthon V, et al. Total knee arthroplasty after high tibial osteotomy: a comparison of opening and closing wedge osteotomy. Int Orthop. 2013;37:427-431.

48. Golovakha ML, Orljanski W, Benedetto KP, et al. Comparison of theoretical fixation stability of three devices employed in medial opening wedge high tibial osteotomy: a finite element analysis. BMC Musculoskelet Disord. 2014;15:230. 
49. Hernigou P, Flouzat Lachaniette C, Delambre J, et al. Full weight bearing and dynamisation with Limmed ${ }^{\circledR}$ locked plate fixation accelerates bone regeneration in the volume of opening wedge high tibial osteotomy. Int Orthop. 2015; 39:1295-1300.

50. Fu D, Li G, Chen K, et al. Comparison of high tibial osteotomy and unicompartmental knee arthroplasty in the treatment of unicompartmental osteoarthritis: a meta-analysis. J Arthroplasty. 2013;28:759-765.

51. Harris JD, McNeilan R, Siston RA, et al. Survival and clinical outcome of isolated high tibial osteotomy and combined biological knee reconstruction. Knee. 2013;20:154-161.

52. Richmond JC. Surgery for osteoarthritis of the knee. Med Clin North Am. 2009;93:213-222.

53. Lobenhoffer P. Importance of osteotomy around to the knee for medial gonarthritis. Indications, technique and results. Orthopade. 2014;43:425-431.

54. Yim JH, Song EK, Seo HY, et al. Comparison of high tibial osteotomy and unicompartmental knee arthroplasty at a minimum follow-up of 3 years. J Arthroplasty. 2013;28:243-247.

55. Soleimanpour J, Elmi A, Jafari MA, et al. Comparison of genu varum treating results using open and closed wedge high tibial osteotomy. Pak J Biol Sci. 2013;16:686-691.

56. Deie M, Hoso T, Shimada N, et al. Differences between opening versus closing high tibial osteotomy on clinical outcomes and gait analysis. Knee. 2014;21:1046-1051.

57. Duivenvoorden T, Brouwer RW, Baan A, et al. Comparison of closing-wedge and opening-wedge high tibial osteotomy for medial compartment osteoarthritis of the knee: a randomized controlled trial with a six-year follow-up. J Bone Joint Surg Am. 2014;96:1425-1432.

58. Brouwer RW, Bierma-Zeinstra SM, van Raaij TM, et al. Osteotomy for medial compartment arthritis of the knee using a closing wedge or an opening wedge controlled by a Puddu plate. A one-year randomised, controlled study. J Bone Joint Surg Br. 2006;88:1454-1459.

59. Bode G, von Heyden J, Pestka J, et al. Prospective 5-year sur- vival rate data following open-wedge valgus high tibial osteotomy. Knee Surg Sports Traumatol Arthrosc. 2015;23:19491955.

60. DeMeo PJ, Johnson EM, Chiang PP, et al. Midterm follow-up of opening-wedge high tibial osteotomy. Am J Sports Med. 2010;38:2077-2084.

61. Faschingbauer M, Nelitz M, Urlaub S, et al. Return to work and sporting activities after high tibial osteotomy. Int Orthop. 2015;39:1527-1534.

62. Bonnin MP, Laurent JR, Zadegan F, et al. Can patients really participate in sport after high tibial osteotomy? Knee Surg Sports Traumatol Arthrosc. 2013;21:64-73.

63. Saito T, Kumagai K, Akamatsu Y, et al. Five- to ten-year outcome following medial opening-wedge high tibial osteotomy with rigid plate fixation in combination with an artificial bone substitute. Bone Joint J. 2014;96-B:339-344.

64. Saragaglia D, Rouchy RC, Krayan A, et al. Return to sports after valgus osteotomy of the knee joint in patients with medial unicompartmental osteoarthritis. Int Orthop. 2014;38:2109-2114.

65. Niemeyer P, Schmal H, Hauschild O, et al. Open-wedge osteotomy using an internal plate fixator in patients with medial-compartment gonarthritis and varus malalignment: 3year results with regard to preoperative arthroscopic and radiographic findings. Arthroscopy. 2010;26:1607-1616.

66. Hernigou P, Roussignol X, Flouzat-Lachaniette $\mathrm{CH}$, et al. Opening wedge tibial osteotomy for large varus deformity with Ceraver resorbable beta tricalcium phosphate wedges. Int Orthop. 2010;34:191-199.

67. Schröter S, Gonser CE, Konstantinidis L, et al. High complication rate after biplanar open wedge high tibial osteotomy stabilized with a new spacer plate (Position HTO plate) without bone substitute. Arthroscopy. 2011;27:644-652.

68. Laprade RF, Spiridonov SI, Nystrom LM, et al. Prospective outcomes of young and middle-aged adults with medial compartment osteoarthritis treated with a proximal tibial opening wedge osteotomy. Arthroscopy. 2012; 28:354-364. 